\title{
Vibrio cholerae 01 epidemic variants in Angola: a retrospective study between 1992 and 2006
}

\section{Romy Valia ${ }^{1+}$, Elisa Taviani ${ }^{1,2+}$, Matteo Spagnoletti ${ }^{3}$, Daniela Ceccarelli ${ }^{4}$, Piero Cappuccinelli ${ }^{5}$ and Mauro M. Colombo ${ }^{1,2 *}$}

' Dipartimento di Biologia e Biotecnologie C. Darwin, Università di Roma Sapienza, Rome, Italy

${ }^{2}$ Centro de Biotecnologia, Universidade E. Mondlane Maputo, Mozambique

${ }^{3}$ Department of Genetics, University College London Genetics Institute, Evolution and Environment, University College London, London, UK

${ }^{4}$ Department of Cell Biology and Molecular Genetics, Maryland Pathogen Research Institute, University of Maryland, College Park, MD, USA

${ }^{5}$ Dipartimento di Scienze Biomediche, Università di Sassari, Sassari, Italy

\section{Edited by:}

Hongyue Dang, Xiamen University, China

\section{Reviewed by:}

Irma N. G. Rivera, University of São Paulo, Brazil

Yael Danin-Poleg, Technion - Israel Institute of Technology, Israel

\section{*Correspondence:}

Mauro M. Colombo, Dipartimento di

Biologia e Biotecnologie Charles

Darwin, Università di Roma

Sapienza, Via dei Sardi 70, 00185

Roma, Italy

e-mail:mauro.colombo@uniroma1.it

${ }^{\dagger}$ These authors have contributed equally to this work.

Cholera is still a major public health concern in many African countries. In Angola, after a decade of absence, cholera reemerged in 1987, spreading throughout the country until 1996, with outbreaks recurring in a seasonal pattern. In 2006 Angola was hit by one of the most severe outbreaks of the last decade, with ca. 240,000 cases reported. We analyzed 21 clinical strains isolated between 1992 and 2006 from several provinces throughout the country: Benguela, Bengo, Luanda, Cuando Cubango, and Cabinda. We used two multiplex PCR assays to investigate discriminatory mobile genetic elements (MGE) [Integrative Conjugative Elements (ICEs), VSP-II, GI12, GI14, GI15, K, and TLC phages] and we compared the profiles obtained with those of different reference $V$. cholerae 01 variants (prototypical, altered, and hybrid), responsible for the ongoing 7th pandemic. We also tested the strains for the presence of specific VSP-II variants and for the presence of a genomic island (GI) (WASA-1), correlated with the transmission of seventh pandemic cholera from Africa to South America. Based on the presence/absence of the analyzed genetic elements, five novel profiles were detected in the epidemic strains circulating in the 1990s. The most frequent profiles, $F$ and $G$, were characterized by the absence of ICEs and the three Gls tested, and the presence of GI WASA-1 and the WASA variant of the VSP-II island. Our results identified unexpected variability within the 1990s epidemic, showing different rearrangements in a dynamic part of the genome not present in the prototypical $V$. cholerae 01 N16961. Moreover the 2006 strains differed from the current pandemic $V$. cholerae $\mathrm{O} 1$ strain. Taken together, our results highlight the role of horizontal gene transfer (HGT) in diversifying the genetic background of $V$. cholerae within a single epidemic.

\section{Keywords: V. cholerae 01, Angola, mobilome, genomic islands, epidemic variants}

\section{INTRODUCTION}

Vibrio cholerae, the etiological agent of cholera, a gastrointestinal infection, has been responsible for seven known pandemics with the seventh pandemic currently occurring. To date, over 200 distinct serogroups have been described, with only serogroups $\mathrm{O} 1$ and $\mathrm{O} 139$ associated with epidemic and pandemic cholera. V. cholerae serogroup O1 strains can be further classified in two biotypes, El Tor and Classical, based on differences in their phenotypic and genotypic traits (Kaper et al., 1995).

During the seventh pandemic several epidemic lineages of $V$. cholerae O1 El Tor have emerged. Most notable was the emergence, in 1992, of a new epidemic serogroup in India and Bangladesh, named O139 or Bengal, which initially displaced the local existing O1 El Tor strains (Faruque et al., 2003). Molecular analyses demonstrated horizontal gene transfer (HGT) as the mechanism by which $V$. cholerae $\mathrm{O} 1$ strains acquired the $\mathrm{O} 139$ surface antigen resulting in a new epidemic serogroup (Bik et al., 1995).
Subsequently, new variants of $V$. cholerae $\mathrm{O} 1$ with features of both the Classical and the El Tor biotypes have been repeatedly isolated in Asia and Africa and are collectively called "Atypical El Tor" strains. This group includes the Matlab types I, II, and III (Nair et al., 2002), Altered El Tor (Nair et al., 2006), Mozambique El Tor (Ansaruzzaman et al., 2004) and Hybrid El Tor strains (Safa et al., 2008).

Recent comparative genomics studies demonstrated that $V$. cholerae $\mathrm{O} 1$ strains circulating during the current seventh pandemic, which include prototypical El Tor strains, Atypical El Tor variants, and the $\mathrm{O} 139$ serogroup, belong to a single phyletic lineage named 7th pandemic clade (Chun et al., 2009). These variants are a result of HGT and slight divergence in strains from a common recent ancestor. Seventh pandemic El Tor strains are in fact characterized by a highly conserved genome background and different combinations of mobile genetic elements (MGE), referred to as the mobilome, such as Integrative Conjugative Elements (ICE), genomic islands (GIs), and prophages (Cho et al., 2010). 
Seventh pandemic $V$. cholerae El Tor clones appear to have disseminated globally from a single source (the Bay of Bengal) in at least three independent waves, all of which reached Africa (Mutreja et al., 2011). Comparative genomic analyses have also suggested that the African continent served as a bridge for the spread of seventh pandemic $V$. cholerae strains from Asia to the Americas. Mutreja et al. (2011) observed that an Angolan strain, isolated in 1989, clustered at the base of the South American clade with a difference of only ten SNPs in the genomic backbone and the presence of two newly observed GIs: a novel variant of VSP-II, the VSP-II WASA (West African-South America), and a new GI named WASA-1 (Mutreja et al., 2011).

Angola is emerging from a 40 years war that ended in 2002 and resulted in significant social and economic disorders. Less than $4 \%$ of the population has access to potable water and adequate health and sanitary services. These conditions resulted in an almost immediate initiation of a cholera epidemic after $V$. cholerae isolates associated with the 7 th cholera pandemic entered the African continent in the early 1970s. This region is now considered an endemic area for cholera (Lam et al., 2010). The most recent outbreak in Angola occurred from February to April 2013, and resulted in 610 cases and 8 deaths (International Society for Infectious Diseases, 2013).

Along with the most recent outbreak, two other major cholera epidemics were reported in Angola in the past 30 years. In April 1987 an epidemic started in the north-east of the country and rapidly spread to all 18 provinces, recurring in a seasonal pattern (Colombo et al., 1993). The outbreak was followed by a 10 years absence of the disease until 2006 when cholera re-emerged in Luanda and rapidly spread throughout the country. The 2006 outbreak was one of the most severe recent cholera epidemics in Africa (World Health Organization, 2007).

We previously demonstrated that the $V$. cholerae $\mathrm{O} 1$ strain responsible for the 2006 Angolan outbreak was an atypical O1 El Tor variant earlier detected only in Asia, and that it was clonally and genetically different from El Tor strains circulating in the 1990s in the same area (Ceccarelli et al., 2011a). Angolan strains from 2006 carried the SXT-related ICEVchAng3, associated with a more narrow multidrug resistance profile compared to the one conferred by plasmid p3iANG harbored by strains from the 1990s (Ceccarelli et al., 2006, 2011a). In light of the new insights into the phylogeny of seventh pandemic $V$. cholerae O1, we intended to reanalyze the Angolan epidemics in order to better understand the epidemiology of cholera in Angola and to confirm its role in bridging Africa and South America during waves of the seventh pandemic. In this study, we screened a collection of Angolan $V$. cholerae $\mathrm{O} 1$ isolates for the presence/absence of MGEs in order to study their collective mobilome and determine possible correlations with the known seventh pandemic variants (Spagnoletti et al., 2012).

\section{MATERIALS AND METHODS V. cholerae STRAINS}

Twenty-one clinical $V$. cholerae strains isolated during the two separate epidemics in Angola in 1996 and 2006 were analyzed (Ceccarelli et al., 2006, 2011a). Five completely sequenced reference strains were used as positive controls (Table 1): V. cholerae
O1 N16961, isolated in India in 1975 (Kaper et al., 1995; Heidelberg et al., 2000); V. cholerae O139 MO10, isolated in India in 1992 (Ramamurthy et al., 1993); V. cholerae O1 CIRS101 (Altered El Tor), isolated in Bangladesh in 2002 (Nair et al., 2006); V. cholerae O1 B33 (Mozambique variant), isolated in Mozambique in 2004 (Ansaruzzaman et al., 2004); V. cholerae O1 MJ1236 (Matlab type I), isolated in Bangladesh in 1994 (Nair et al., 2002). V. cholerae strains analyzed in this work are listed in Table 1. Bacterial strains were routinely grown on LB Agar at $37^{\circ} \mathrm{C}$ for $16-18 \mathrm{~h}$ with appropriate antibiotic selection and were maintained at $-80^{\circ} \mathrm{C}$ in $\mathrm{LB}$ broth containing $15 \%$ (vol/vol) glycerol.

\section{PRIMERS AND PCR CONDITIONS}

Genomic DNA was prepared with a Wizard ${ }^{\circledR}$ Genomic DNA Purification Kit (Promega), according to manufacturer's instructions. PCR was performed in $50 \mu \mathrm{l}$ reaction mix containing $1 \mathrm{U}$ of GoTaq DNA polymerase (Promega) and $50 \mathrm{ng} / \mu \mathrm{L}$ DNA. Amplification was performed in an automated thermocycler (BioRad MJ-Mini Personal Thermal Cycler). Oligonucleotides and PCR conditions for the double multiplex PCR were optimized as previously described (Spagnoletti et al., 2012).

WASA-1 and VSP-2 WASA presence was investigated with new primer pairs designed using Angolan strain V. cholerae O1 El Tor A5 (Mutreja et al., 2011) as reference (Accession no ERS013245): (i) wasa1F ( $5^{\prime}$-CCAAAGCAGAGAGACGCA- 3$)^{\prime}$, and wasa1R $\left(5^{\prime}\right.$ GTTCTCACCTTCTTCCGCA-3') giving an amplicon size of 464 bp; (ii) VSP2wasaF (5'-GTGCTGTATTTGGTTTGATGGGT$3^{\prime}$ ), and VSP2 wasaR ( $5^{\prime}$-GATAGTGGTTTCGCTGAGATTGT-3'), resulting in an amplicon size of $438 \mathrm{bp}$. Oligonucleotides were obtained from PRIMM srl (Milano, Italy).

\section{RESULTS}

Of the 21 strains analyzed during this study, 19 were isolated between 1992 and 1996 from the provinces of Benguela, Bengo, Luanda, Cuando Cubango (South-east), and the Cabinda enclave (North-west) (Figure 1). Two clinical strains isolated in Luanda in 2006 were also included in this study. All the strains were subjected to the double multiplex PCR screening and their amplicon profiles compared with the reference strains (Table 1, profiles A-J). WASA-1 analysis was not included in the profile definition but was analyzed separately to be consistent with the original double Multiplex PCRs approach (Spagnoletti et al., 2012). It is important to note that primers included in Multiplex 1 (VSPIIintF/R and VSPIIcutF/R) and used to discriminate prototypical VSP-II from other variants (Spagnoletti et al., 2012) gave no amplification for both the deleted CIRS-like VSP-II island and the WASA VSP-II. Therefore, rearrangements in the non-prototypical VSP-II islands were further tested with the VSP2 wasaF/R primer pair. The five reference strains showed unique expected profiles (Spagnoletti et al., 2012), as listed in Table 1.

\section{ANALYSIS OF V. cholerae 01 EPIDEMIC STRAINS ISOLATED BETWEEN 1992 AND 1996}

Six clinical strains isolated in 1992 and 1993 in Luanda showed two different mobilome profiles. Profile F (four strains from 1992 and one strain from 1993) is characterized by the presence of a 
Table 1 | V. cholerae strains analyzed in this study.

\begin{tabular}{|c|c|c|c|c|c|c|c|c|c|c|}
\hline \multirow[t]{2}{*}{ Strain } & \multirow[t]{2}{*}{ Isolation } & \multicolumn{2}{|c|}{ Multiplex 1} & \multicolumn{5}{|c|}{ Multiplex 2} & \multirow[t]{2}{*}{ Profile } & \multirow[t]{2}{*}{ Wasa-1 } \\
\hline & & ICE & VSP-IIa & phage $K$ & Gl-12 & GI-14 & Gl-15 & TLC & & \\
\hline \multicolumn{11}{|l|}{ REFERENCE } \\
\hline V. cholerae O1 N16961 & 1975, India & - & + & - & - & - & - & + & A & - \\
\hline V. cholerae 01 CIRS101 & 2002, Bangladesh & ICEVchlnd5 & non-prototypical & - & - & - & - & + & B & - \\
\hline V. cholerae O1 B33 & 2004, Mozambique & ICEVchMoz10 $10^{\mathrm{b}}$ & + & + & - & + & + & - & $\mathrm{C}$ & - \\
\hline V. cholerae O1 MJ1236 & 1994, Bangladesh & ICEVchBangb & + & + & + & + & - & - & D & - \\
\hline V. cholerae O139 M010 & 1992, India & $\mathrm{SXT}$ & + & + & - & - & - & + & $E$ & - \\
\hline V. cholerae $\mathrm{O} 1 \mathrm{~A} 5^{\mathrm{C}}$ & 1989, Angola & - & WASA & $\mathrm{n} / \mathrm{a}$ & $\mathrm{n} / \mathrm{a}$ & $\mathrm{n} / \mathrm{a}$ & $\mathrm{n} / \mathrm{a}$ & $\mathrm{n} / \mathrm{a}$ & $\mathrm{n} / \mathrm{a}$ & + \\
\hline \multicolumn{11}{|l|}{ ANGOLA } \\
\hline V. cholerae 01573 & 1992, Luanda & - & WASA & + & - & - & - & + & $\mathrm{F}$ & + \\
\hline V. cholerae 01588 & 1992, Luanda & - & WASA & + & - & - & - & + & $\mathrm{F}$ & + \\
\hline V. cholerae 01583 & 1992, Luanda & - & WASA & + & - & - & - & + & $\mathrm{F}$ & + \\
\hline V. cholerae 01 611A & 1992, Luanda & - & WASA & + & - & - & - & + & $\mathrm{F}$ & + \\
\hline V. cholerae 01582 & 1992, Luanda & - & WASA & - & - & - & - & + & $\mathrm{G}$ & + \\
\hline V. cholerae 01663 & 1993, Luanda & - & WASA & + & - & - & - & + & $\mathrm{F}$ & + \\
\hline V. cholerae 011349 & 1994, Caxito (Bengo) & - & WASA & - & - & - & - & + & $\mathrm{G}$ & + \\
\hline V. cholerae 011350 & 1994, Luanda & - & WASA & + & - & - & - & + & $\mathrm{F}$ & - \\
\hline V. cholerae 011356 & 1994, Cuando Cubango & - & WASA & + & - & - & - & + & $\mathrm{F}$ & - \\
\hline V. cholerae 011382 & 1994, Cuando Cubango & - & WASA & + & - & - & - & - & $\mathrm{H}$ & - \\
\hline V. cholerae 01819 & 1994, Dondo, Cuanza Norte & - & WASA & - & - & - & - & + & $\mathrm{G}$ & - \\
\hline V. cholerae 011354 & 1994, Dondo, Cuanza Norte & - & WASA & - & - & - & - & + & G & + \\
\hline V. cholerae 011383 & 1994, Benguela & - & WASA & + & - & - & - & + & $\mathrm{F}$ & - \\
\hline V. cholerae 011357 & 1995, Cazenga, Luanda & - & WASA & + & - & - & - & + & $\mathrm{F}$ & + \\
\hline V. cholerae 011359 & 1995, Sambizanga Luanda & - & WASA & + & - & - & - & + & $\mathrm{F}$ & + \\
\hline V. cholerae 011358 & 1995, Tala Hady Luanda & - & WASA & - & - & - & - & - & 1 & + \\
\hline V. cholerae 011360 & 1995, Cazenga, Luanda & - & WASA & - & - & - & - & - & I & - \\
\hline V. cholerae 011361 & 1995, Cabinda & - & WASA & + & - & - & - & + & $\mathrm{F}$ & - \\
\hline V. cholerae 01908 & 1996, Cabinda & - & WASA & - & - & - & - & - & I & - \\
\hline V. cholerae O1 VC175 & 2006, Luanda & ICEVchAng3 & + & - & - & - & - & + & $J$ & - \\
\hline V. cholerae O1 VC189 & 2006, Luanda & ICEVchAng3 & + & - & - & - & - & + & $J$ & - \\
\hline
\end{tabular}

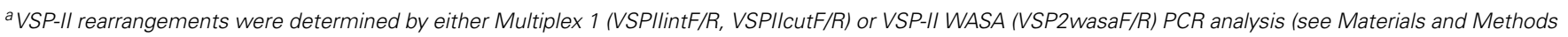
for further details).

b Sibling ICEs (Ceccarelli et al., 2011b).

c Information about this strain is derived from Mutreja et al. (2011).

non-prototypical VSP-II, and phages K and TLC; and profile G (strain 582 from 1992) carries the non-prototypical VSP-II and the TLC phage. All non-prototypical VSP-II islands were confirmed as carrying WASA insertion. Additionally, all six strains showed the expected amplification for WASA-1 GI.

Seven clinical $V$. cholerae $\mathrm{O} 1$ strains isolated in five different provinces of the country in 1994 (Figure 1) gave three profiles (Table 1). Similar to the 1992 and 1993 strains isolated from Luanda, the two most common profiles were $\mathrm{F}$ and G. Profile F strains were isolated from Luanda (isolate 1350), Cuando Cubango (isolate 1356), and Benguela (isolate 1383). Profile $G$ strains were isolated from Bengo province (isolate 1349), and Dondo (Cuanza Norte) (isolates 819 and 1354). A previously unobserved profile $\mathrm{H}$ (non-prototypical VSP-II and phage K) was observed in strain 1382 isolated in Cuando Cubango. All non-prototypical VSP-II islands were confirmed as carrying the WASA insertion. V. cholerae strains 1349 and 1354 gave a positive amplification for WASA-1 GI while the remaining five strains gave negative amplification for this cluster.

Five clinical $V$. cholerae O1 strains from 1995 and one from 1996 were isolated in the provinces of Luanda and Cabinda (Figure 1). Strains isolated in four different municipalities of the Luanda province in 1995 showed two profiles: profile F (strains 1357 and 1359), and profile I (strains 1358 and 1360) in which the sole non-prototypical variant of VSP-II was amplified. The two strains isolated in the Cabinda enclave showed profiles $\mathrm{F}$ and I in 1995 (strain 1361) and 1996 (strain 908), respectively. All non-prototypical VSP-II islands were confirmed as carrying the WASA insertion. V. cholerae strains 1357, 1358, and 1359 from Luanda gave a positive amplification for the WASA-1 GI while the remaining 3 strains (isolates 1360, 1361, and 908 from Luanda and the Cabinda enclave, respectively) were negative for this cluster. 


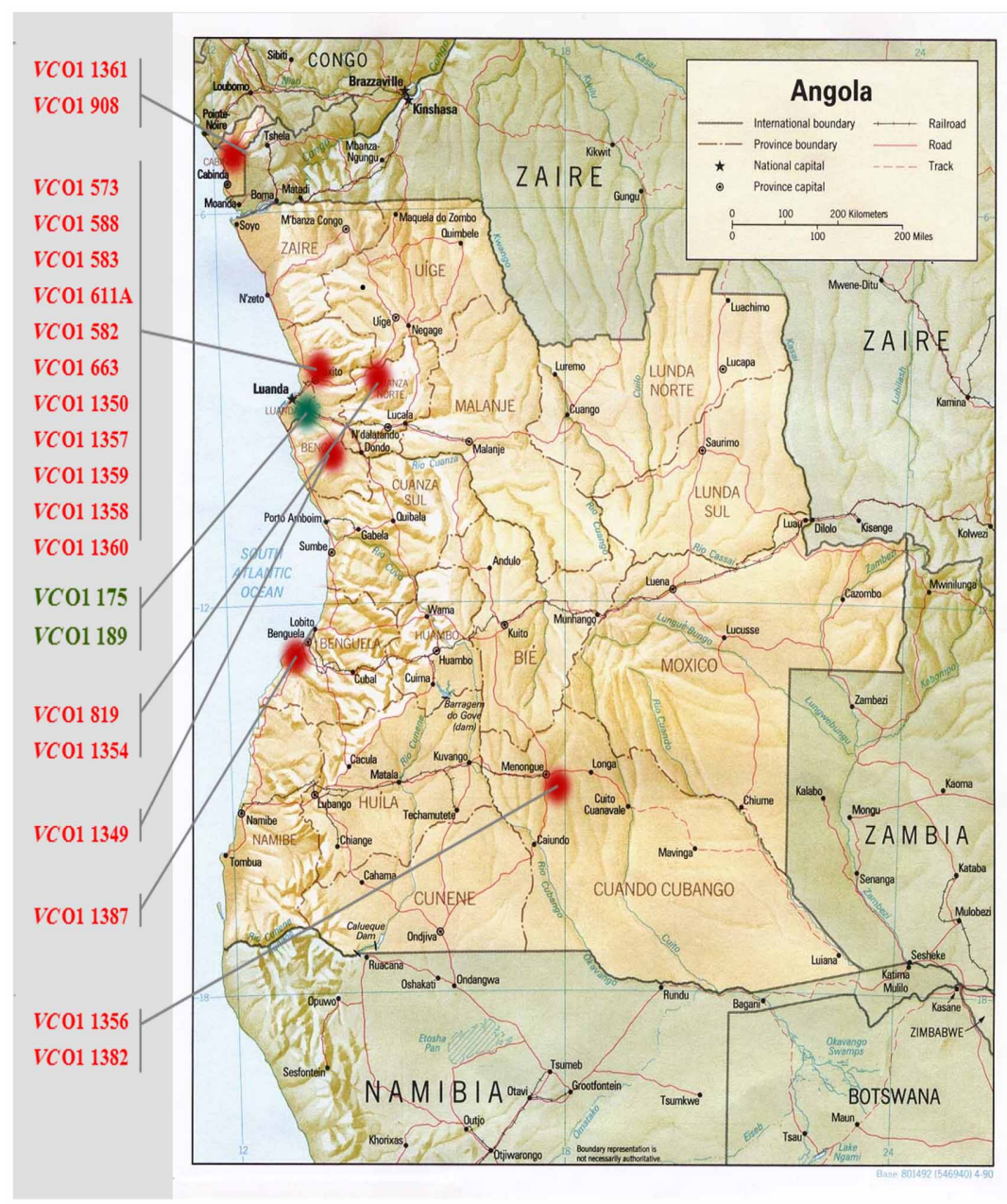

FIGURE 1 | Map of Angola showing the locations of $\boldsymbol{V}$. cholerae 01 isolation in this analysis. Strains isolated during the $1992-1996$ epidemics are indicated in red, strains isolated during 2006 epidemics are indicated in green.

\section{ANALYSIS OF V. cholerae 01 EPIDEMIC STRAINS ISOLATED IN 2006}

As anticipated by our previous study (Ceccarelli et al., 2011a), the two $V$. cholerae $\mathrm{O} 1$ strains isolated in Luanda during the 2006 epidemic, shared a common and previously unobserved mobilome profile J (Table 1). These strains harbored the prototypical intact variant of VSP-II, an ICE element (ICEVchAng3, sibling of ICEVchInd5), and TLC. Both were negative for the presence of WASA-1 and VSP-II WASA genetic clusters.

\section{DISCUSSION}

This retrospective study is based on a collection of Vibrio cholerae O1 clinical strains, isolated from 1992 to 2006 from different provinces in Angola (Figure 1 and Table 1). Despite encouragement from local national health authorities, it was not possible to isolate strains over a broader temporal and geographic scale due to security and logistic constraints caused by socio-economic conditions. Thus, we are aware of the potential spatial and temporal biases inherent in this set of strains. Half of our isolates were recovered from cases in Luanda, and all but two isolates were recovered between 1992 and 1996 . Nevertheless, we believe the strains presented in this study represent an important source of data depicting the presence of a variable population of $V$. cholerae $\mathrm{O} 1$ strains in the country.

The epidemiology of $V$. cholerae $\mathrm{O} 1$ in Africa can be explained by multiple introductions of cholera from endemic regions of Asia, with the first introduction following the early dissemination of seventh pandemic in the 1970s (Kaper et al., 1995). In our previous analysis we hypothesized that $V$. cholerae $\mathrm{O} 1$ strains responsible for the first epidemic (1987-1996) in Angola are progeny of the prototypical strain represented by $V$. cholerae O1 El Tor N16961, with the only difference being the presence of the p3iANG plasmid (Ceccarelli et al., 2011a). However, our current study revealed that all $V$. cholerae O1 strains from 1987 to 1996 harbored the WASA variant of the VSP-II GI, and 10 of these carried also the WASA-1 GI. Therefore, these strains differed in MGE content from V. cholerae N16961 (profile A). An even greater level of diversity was identified in these 
strains when tested for the presence of the K and TLC phages. These analyses resulted in the assignment of four distinct profiles (F-I) among strains isolated between 1987 and 1996 in Angola.

The two strains isolated in 2006 showed a mobilome profile highly similar to that of the altered El Tor V. cholerae O1 strain CIRS101, including the presence of ICEVchAng3, an ICE element of the SXT/R391 family sibling with ICEVchInd5, the most widespread ICE present in V. cholerae O1 CIRS101-like strains isolated in the Indian Subcontinent and worldwide (Ceccarelli et al., 2011a,b,c). Again, significant differences between the 2006 Angolan strains and V. cholerae CIRS101 were identified in the VSP-II island. The 2006 Angolan strains encoded the prototypical VSP-II, while many currently isolated pandemic $V$. cholerae O1 CIRS101-like strains isolated worldwide after 2001 encode a deletion in VSP-II (Taviani et al., 2010). These results suggest that epidemic strains circulating in Angola in 2006 harbored a genetic background with features similar to strains responsible for the first (profile A) and the third (profile B) pandemic waves.

All strains in this analysis were negative for GIs-12, -14 , and -15 , which are specific GIs of atypical El Tor strains similar to V. cholerae O1 MJ1236 and B33 (Grim et al., 2010). The latter was isolated in Beira, Mozambique, in 2004, but it was recently demonstrated to be present in Austral Africa since the 1990s (Spagnoletti et al., 2012). By testing these three GIs as molecular markers, our analysis suggests that the Angolan epidemics were not directly influenced by contemporary eastern Africa epidemics, which are thought to have been initiated by the second pandemic wave (Mutreja et al., 2011).

Our analysis revealed the presence of the WASA-1 GI in all $V$. cholerae O1 strains from 1992, whereas the same element was absent in recently recovered isolates. The VSP-II WASA variant was instead present in all $V$. cholerae $\mathrm{O} 1$ strains. The presence of the WASA clusters in Angolan $V$. cholerae O1 strains confirmed the Mutreja's hypothesis, based only on one isolate from 1989, that the transmission of the seventh pandemic to South America may have occurred via the African continent and, specifically, through Angola (Mutreja et al., 2011). Furthermore, it suggests that transmission of a strain which encoded both VSPII-WASA and WASA-1 is likely to have occurred during the 1980s, as previously reported (Lam et al., 2010; Mutreja et al., 2011).

In conclusion, the history of the Angolan epidemic adds new and valuable information to evolutionary history of $V$. cholerae O1 El Tor within the seventh pandemic. At the time-scale presented here and elsewhere, this evolution occurs mainly via lateral gene transfer events driven by environmental factors. Interestingly, none of the Angolan strains showed a mobilome profile similar to those of the representative 7 th pandemic strains. The variability within these strains was greater than expected, as strains isolated within the same epidemic were shown to harbor different constellations of mobile elements. A similar variability was previously observed in a collection of strains isolated in Mozambique in 1997-1998 (Spagnoletti et al., 2012), thus reflecting frequent HGT dynamics similar to the Angolan epidemic.

\section{ACKNOWLEDGMENTS}

This work was funded by Ministry of Foreign Affair-DGCS, Italy, Ministero dell'Istruzione, dell'Università e della RicercaItaly (PRIN). Matteo Spagnoletti is supported by a fellowship from Istituto Pasteur-Cenci Bolognetti Foundation, Italy. The English revision was carried out by Bradd J. Haley.

\section{REFERENCES}

Ansaruzzaman, M., Bhuiyan, N., Nair, B., Sack, D., Lucas, M., Deen, J., et al. (2004). Cholera in Mozambique, variant of Vibrio cholerae. Emerg. Infect. Dis. 10, 2057-2059. doi: 10.3201/eid1011.040682

Bik, E. M., Bunschoten, A. E., Gouw, R. D., and Mooi, F. R. (1995). Genesis of the novel epidemic Vibrio cholerae O139 strain: evidence for horizontal transfer of genes involved in polysaccharide synthesis. EMBO J. 14, 209-216.

Ceccarelli, D., Salvia, A. M., Sami, J., Cappuccinelli, P., and Colombo, M. M. (2006). New cluster of plasmid-located class 1 integrons in Vibrio cholerae $\mathrm{O} 1$ and a $d$ frA15 cassette-containing integron in Vibrio parahaemolyticus isolated in Angola. Antimicrob. Agents Chemother. 50, 2493-2499. doi: 10.1128/AAC.01310-05

Ceccarelli, D., Spagnoletti, M., Bacciu, D., Cappuccinelli, P., and Colombo, M. M. (2011a). New, V. cholerae atypical El Tor variant emerged during the 2006 epidemic outbreak in Angola. BMC Microbiol. 11:130. doi: 10.1186/1471-218011-130

Ceccarelli, D., Spagnoletti, M., Bacciu, D., Danin-Poleg, Y., Mendiratta, D., Kashi, Y., et al. (2011b). ICEVchInd5 is prevalent in epidemic Vibrio cholerae O1 El Tor strains isolated in India. Int. J. Med. Microbiol. 301, 318-324. doi: 10.1016/j.ijmm.2010.11.005

Ceccarelli, D., Spagnoletti, M., Cappuccinelli, P., Burrus, V., and Colombo, M. M. (2011c). Origin of Vibrio cholerae in Haiti. Lancet Infect. Dis. 11, 260. doi: 10.1016/S1473-3099(11)70078-0

Cho, Y. J., Yi, H., Lee, J. H., Kim, D. W., and Chun, J. (2010). Genomic evolution of Vibrio cholerae. Curr. Opin. Microbiol. 13, 646-651. doi: 10.1016/j.mib.2010.08.007

Chun, J., Grim, C. J., Hasan, N. A., Lee, J. H., Choi, S. Y., Haley, B. J., et al. (2009). Comparative genomics reveals mechanism for short-term and longterm clonal transitions in pandemic Vibrio cholerae. Proc. Natl. Acad. Sci. U.S.A. 106, 15442-15447. doi: 10.1073/pnas.0907787106

Colombo, M. M., Francisco, M., Ferreira, B. D., Rubino, S., and Cappuccinelli, P. (1993). The early stage of the recurrent cholera epidemic in Luanda, Angola. Eur. J. Epidemiol. 9, 563-565. doi: 10.1007/BF00209537

Faruque, S. M., Sack, D. A., Sack, R. B., Colwell, R. R., Takeda, Y., and Nair, G. B. (2003). Emergence and evolution of Vibrio cholerae O139. Proc. Natl. Acad. Sci. U.S.A. 100, 1304-1309. doi: 10.1073/pnas.0337468100

Grim, C. J., Hasan, N. A., Taviani, E., Haley, B., Chun, J., Brettin, T. S., et al. (2010). Genome sequence of hybrid V. cholerae O1 MJ-1236, B-33 and CIRS101 and comparative genomics with V. cholerae. J. Bacteriol. 192, 3524-3533. doi: 10.1128/JB.00040-10

Heidelberg, J. F., Eisen, J. A., Nelson, W. C., Clayton, R. A., Gwinn, M. L., Dodson, R. J., et al. (2000). DNA sequence of both chromosomes of the cholera pathogen Vibrio cholerae. Nature 406, 477-483. doi: 10.1038/35020000

International Society for Infectious Diseases. (2013). Cholera, Diarrhea and Dysentery Update (13): Africa, Asia [Online]. Available online at: http://www. promedmail.org. (Accessed: March 19, 2013).

Kaper, J. B., Morris, Jr. J. G., and Levine, M. M. (1995). Cholera. Clin. Microbiol. Rev. 8, 48-86.

Lam, C., Octavia, S., Reeves, P., Wang, L., and Lan, R. (2010). Evolution of seventh cholera pandemic and origin of 1991 epidemic, Latin America. Emerg. Infect. Dis. 16, 1130-1132. doi: 10.3201/eid1607.100131

Mutreja, A., Kim, D. W., Thomson, N. R., Connor, T. R., Lee, J. H., Kariuki, S., et al. (2011). Evidence for several waves of global transmission in the seventh cholera pandemic. Nature 477, 462-465. doi: 10.1038/nature10392

Nair, G. B., Faruque, S. M., Bhuiyan, N. A., Kamruzzaman, M., Siddique, A. K., and Sack, D. A. (2002). New variants of Vibrio cholerae O1 biotype El Tor with attributes of the classical biotype from hospitalized patients with acute diarrhea in Bangladesh. J. Clin. Microbiol. 40, 3296-3299. doi: 10.1128/JCM.40.9.32963299.2002 
Nair, G. B., Qadri, F., Holmgren, J., Svennerholm, A.-M., Safa, A., Bhuiyan, N. A., et al. (2006). Cholera due to altered El Tor strains of Vibrio cholerae O1 in Bangladesh. J. Clin. Microbiol. 44, 4211-4213. doi: 10.1128/JCM.01304-06

Ramamurthy, T., Garg, S., Sharma, R., Bhattacharya, S. K., Nair, G. B., Shimada, T., et al. (1993). Emergence of a novel strain of Vibrio cholerae with epidemic potential in Southern and Eastern India. Lancet 341, 703-704. doi: 10.1016/0140-6736(93)90480-5

Safa, A., Sultana, J., Cam, P. D., Mwansa, J. C., and Kong, R. Y. C. (2008). Vibrio cholerae O1 Hybrid El Tor Strains, Asia and Africa. Emerg. Infect. Dis. 14, 987-988. doi: 10.3201/eid1406.080129

Spagnoletti, M., Ceccarelli, D., and Colombo, M. M. (2012). Rapid detection by multiplex PCR of Genomic Islands, prophages and Integrative Conjugative Elements in V.cholerae 7th pandemic variants. J. Microbiol. Methods 88, 98-102. doi: 10.1016/j.mimet.2011.10.017

Taviani, E., Grim, C. J., Choi, J., Chun, J., Haley, B., Hasan, N. A., et al. (2010). Discovery of novel Vibrio cholerae VSP-II genomic islands using comparative genomic analysis. FEMS Microbiol. Lett. 308, 130-137. doi: 10.1111/j.15746968.2010.02008.x

World Health Organization. (2007). Cholera 2006. Wkly. Epidemiol. Rec. 31, 273-284. Available online at: http://www.who.int/wer/2007/wer8231.pdf
Conflict of Interest Statement: The authors declare that the research was conducted in the absence of any commercial or financial relationships that could be construed as a potential conflict of interest.

Received: 04 October 2013; paper pending published: 24 October 2013; accepted: 07 November 2013; published online: 28 November 2013.

Citation: Valia R, Taviani E, Spagnoletti M, Ceccarelli D, Cappuccinelli $P$ and Colombo MM (2013) Vibrio cholerae O1 epidemic variants in Angola: a retrospective study between 1992 and 2006. Front. Microbiol. 4:354. doi: 10.3389/fmicb.2013.00354 This article was submitted to Aquatic Microbiology, a section of the journal Frontiers in Microbiology.

Copyright (C) 2013 Valia, Taviani, Spagnoletti, Ceccarelli, Cappuccinelli and Colombo. This is an open-access article distributed under the terms of the Creative Commons Attribution License (CCBY). The use, distribution or reproduction in other forums is permitted, provided the original author(s) or licensor are credited and that the original publication in this journal is cited, in accordance with accepted academic practice. No use, distribution or reproduction is permitted which does not comply with these terms. 